\title{
Research of improved particle swarm algorithm based on apparel intelligent marker making
}

\author{
HUANG Can-yi \\ Business and Information Department \\ Quanzhou Normal University \\ Quanzhou, China \\ yidyj126@sina.com
}

\begin{abstract}
The intelligent algorithm of apparel marker making is the core technology of apparel marker making, this paper brings forward an improved particle swarm algorithm at the base of analyzing the merit and demerit of genetic algorithm, simulated annealing algorithm and particle swarm optimization algorithm, furthermore, a demonstration test is done by $\mathrm{C}++$ under the environment of VS2005. The result indicates the improved particle swarm algorithm can achieve ideal material utilization.
\end{abstract}

Keywords-apparel intelligent marker making; genetic algorithm; simulated annealing algorithm; improved particle swarm algorithm

\section{INTRODUCION}

Garment marker making technology is one of the important technologies of clothing enterprises, the operation time of marker process and the utilization ratio of maker making directly affect the enterprise's cost, profit, product competition ability and economic benefits. Marker making technology has been used as the most important way to raise the material utilization ratio, reduce the material loss and production cost for garment manufacturing enterprises in the production process. Therefore, a set of intelligent and efficient marker making technology can not only reduce the staff's working strength, but also greatly improve the producing speed and the utilization ratio of material, and but also bring considerable economic benefit to enterprises.

With the development and popularization of garment CAD software, the core technology of garment CAD, the intelligent marker algorithm has been improved also. The optimization algorithms which can solve the maker problem conclude artificial neural network, genetic algorithm, heuristic algorithm and simulated annealing algorithm. Therein, the artificial neural network is more difficult to solve optimal marker problem, as it's not suitable for non rectangular pieces; and as genetic algorithm has no special restrictions to its required problem, and more facilitate for organically integration of rectangular parts graphic arithmetic and genetic algorithm, so there are many solutions can solve the optimal marker problem. But the genetic algorithm has limitation which we call the premature phenomenon, and its local searching ability is poor; simulated annealing algorithm has strong overall search ability. In a word, marker making problem is a typical NP complete problem, namely the time complexity of finding the solution is in exponential order. In the relatively large size of the problem, the loss of viability often occurs because of the increasing computing time and, so it is necessary to find other feasible scheme. This paper introduces a method that based on particle swarm algorithm, and does experiment at the same time analysis its effectiveness.

\section{INTRODUCTION FOR GARMENT INTELLIGENT MARKER MAKING ALGORITHM}

Garment marker making problem belongs to the two-dimensional irregular maker making problem, two-dimensional irregular marker making problem refers to the length and width direction, and so two-dimensional marker making problem can be described as: a series of pending pieces $\mathrm{P} 1, \mathrm{P} 2 \ldots \mathrm{Pn}$. Put they reasonably in raw material $\mathrm{P}$, and make the raw material utilization rate as higher as possible, at the same time have to meet the following conditions [1]:

- Piand $\mathrm{Pj}$ will not overlap each other;i,j=1, $2, \ldots$;

- $\quad$ Piand $P j$ must be within the limits of $P ; i, j=1,2, \ldots n$;

- Meet the technique requirements.

\section{A. Genetic algorithm}

Genetic algorithm is a kind of highly parallel, randomized, adaptive searching algorithm which using the development of the experience of natural selection from biological and evolutionary mechanisms. Due to its robustness, it's particularly suited to deal with the complicated and non-linear problems that traditional search algorithms are hard to solve [2].Genetic algorithm develops from Darwin's theory of evolution thought, genetic algorithm uses a natural evolutionary model, according to the "struggle for existence" and "survival of the fittest" principle, through reproduction, crossover, mutation, so it can solve the problem from the initial solution then step by step and approach the optimal solution [3, 4].Early garment CAD algorithms mainly use this algorithm, but for the effect is not so good when doing complex marker making .

\section{B. simulated annealing algorithm}

The concept of simulated annealing mainly comes from a case that when the solid is heated to a certain temperature, solid molecular structure will be dispersed and collapse into the liquid structure, and then if the cooling process is controlled, when completely cooled and the molecules in the liquid structure transit to solid structure, the solid can be rearranged into the desired steady state. When in a local 
optimal solution, simulated annealing algorithm will through reheating action, by a random process, so the algorithm can jump out of local optimal, and have the opportunity to reach another optimal solution. At present, many domestic and foreign garment marker making systems are based on simulated annealing algorithm as their core, but its stability is not very good.

\section{Particle swarm algorithm}

Particle swarm algorithm, an evolutionary computation method that first proposed by Eberhart and Kennedy in 1995. Particle swarm optimization algorithm origins from the simulation of birds foraging behavior, with its artificial life theory as theoretical background, is an optimization algorithm based on swarm intelligence. Particle swarm optimization algorithm, optimization of each feasible solution can be regarded as a bird in the search space, which is a particle. Particle swarm algorithm can be used to solve the optimization problem such as function optimization, system identification and pattern recognition. As a result of the procedure is very simple, few parameters are needed to adjust, so in recent years there has been a research upsurge and resulting in a large number of research results, and has begun in some application areas [5].The algorithm has been widely used by many well-known brands of intelligent garment marker making system, but the performance remains to be improved.

\section{IMPROVED PARTICLE SWARM ALGORITHM}

\section{A. The improvement of particle swarm algorithm}

Suppose there are $\mathrm{m}$ particles in the $\mathrm{n}$-dimensional search space, The first $i$ particle's current position is expressed as $X_{\mathrm{i}}=\left\{\mathrm{X}_{\mathrm{i} 1}, \mathrm{X}_{\mathrm{i} 2} \ldots, \mathrm{X}_{\mathrm{in}}\right\}$, The current speed is expressed as $\mathrm{V}_{\mathrm{i}}=\left\{\mathrm{v}_{\mathrm{i} 1}, \mathrm{v}_{\mathrm{i} 2}, \ldots, \mathrm{v}_{\mathrm{in}}\right\}$, the best position particle $\mathrm{i}$ through is expressed as $\mathrm{X}_{\mathrm{iBest}}=\left\{\mathrm{x}_{\mathrm{ilB}}, \mathrm{X}_{\mathrm{i} 2 \mathrm{~B}}, \ldots, \mathrm{x}_{\mathrm{inB}}\right\}$, the best position the whole particle swarm through is expressed as $\mathrm{X}_{\mathrm{Best}}=\left\{\mathrm{x}_{1 \mathrm{~B}}, \mathrm{X}_{2 \mathrm{~B}}, \ldots, \mathrm{X}_{\mathrm{nB}}\right\}$, and then the basic particle swarm algorithm formula is as follows:

$$
\begin{aligned}
& v_{i j}=v_{i j}+c_{1} \times \operatorname{rand}_{1} 0 \times\left(x_{i j B}-x_{i j}\right)+c_{2} \times \operatorname{rand}_{2} 0 \times\left(x_{i B}-x_{i j}\right) \\
& x_{i j}=x_{i j}+v_{i j}
\end{aligned}
$$

Among them, C1, C2 represents learning factor, rand $_{1}$ () and $\operatorname{rand}_{2}()$ is random number between 0 to $1, \mathrm{j}$ represents the first $\mathrm{j}$ dimension.

Many experiments show that, although the basic particle swarm optimization algorithm premature convergence speed is very quick, but the local search capability is not strong enough, then cause later convergence speed is not ideal, and accuracy is not high too. In order to improve the basic particle swarm optimization algorithm's local search ability, this study introduces inertia weight $\mathrm{W}$ for formula (1), inertia weight $\mathrm{W}$ is a very important parameter for improved particle swarm optimization. It directly influences the global and local search ability of particle swarm optimization algorithm, thus putting formula (1) into:

$v_{i j}=w \times v_{i j}+c_{1} \times \operatorname{rand}_{1} 0 \times\left(x_{i j B}-x_{i j}\right)+c_{2} \times$ rand $_{2} 0 \times\left(x_{i B}-x_{i j}\right)$

B. The general steps of improved particle swarm

\section{optimization algorithm}

Particle swarm algorithm overall steps are summed up as follows:

1) Initializing particle swarm algorithm parameters, such as iterative times, particle size, learning factor, inertia weight $\mathrm{W}$ and other related parameters.

2) Assign a value to each variable

a) The whole particle swarm is indicated by the vector $<$ psolndividual $>$ swarm;

b) Randomly generate initial particle "swarm", determine the current position of each particle indicated by "swarm[i].currentPos";

c) Determine each particle's adjusted speed "swarm[i].speed";

d) Calculate each particle's fitness in initial particle swarm, and then assigned corresponding variable "swarm[i].fitness" to each particle;

e) Initialize each particle's locally optimal location of swarm[i].bestPos for swarm[i].currentpos, the local best fitness value of swarm[i].bestFitness for swarm[i].fitness; initialize the global best position of psoGbest.gBestpos for swarm[0].currentPos.

Among them, i presents the first i scheme of, in other word, it's particle $i, j$ presents the first $j$ waiting piece in marker making scheme, $0 \leq \mathrm{i}<\mathrm{M}, 0 \leq \mathrm{j}<\mathrm{N}$, ;

3) Initialize the number of iterations counter

4) Update local best position of each particle experiences. Traverse the particle swarm, if swarm [i].bestFitness $<$ swarm[i].fitness, then swarm[i].fitness is assigned to swarm [i].bestFitness, at the same time, the local optimal location swarm[i].bestPos is updated to swarm[i].currentPos. Otherwise, do not update.

5) Updating the particle swarm's global best position. Traverse the particle swarm, if PsoGbest.fitness $<$ swarm[i].fitness, then swarm[i].fitness is assigned to psoGbest.fitness, at the same time, the global best position psoGbest.gBestpos is updated to swarm[i].bestpos. Otherwise, do not update;

6) For improved particle swarm optimization algorithm that based on neighborhood mutation, after iterative number counter reaches a detection time, recording the fitness value when the particle swarm through the optimal position, if the fitness values of 10 consecutive particles swarm through the optimal position are the same and less than 0.68 , then randomly select particle $i$ in current particle swarm, through neighborhood variation "swarm[i].currentpos" on its displacement and get newPos, randomly generated the temperature parameter between 0.001 to100, if newPos $\leq$ swarm[i].fitness, then swarm[i].currentPos $=$ newPos, Otherwise, generates a random number, $0 \leq \varepsilon<1$, then judge the value of $\varepsilon$, if

$$
\varepsilon<\exp \left(-\frac{f\left(\text { swarm }[i]_{\text {new }}\right)-f(\text { swarm }[i])}{\mathrm{t}}\right)
$$

Then swarm $[\mathrm{i}]=$ newPos is accepted;

7) Adjusting each particle's speed.

8) Adjusting each particle's location. 
9) Calculating each particle's fitness function value.

10) On the strategy of decreasing inertia weight, according to formula (2) and (3) to adjust the inertia weight $\mathrm{W}$.

11) Determine whether a termination condition is satisfied, if not satisfied, then the number of iterations counter plus 1 , and turn (4); If the termination condition is satisfied, output current particle swarm's best experience position, algorithm end.

\section{MARKER MAKING EXPERIMENTS AND RESULTS ANALYSIS}

A. Condition of marker making experiments

Experimental environment: one PC, hardware configuration of CPU: Pentium4, frequency: $3.0 \mathrm{GHz}$, memory: 1G.Software for the Windows XP Professional operating system, all the algorithms are achieved by $\mathrm{C}++$ based on the platform of vs2005. Considering the apparel styles are complex and changeable, resulting in the clothing plates also has the uncertain shape, so this study cited random generation of two-dimensional irregular graphics algorithm to generate irregular apparel plates, the experiment adopts a $450 \mathrm{~cm} \times 500 \mathrm{~cm}$ fabric, randomly generates 100 irregular apparel plates. Experimental interface is shown in figure 1.

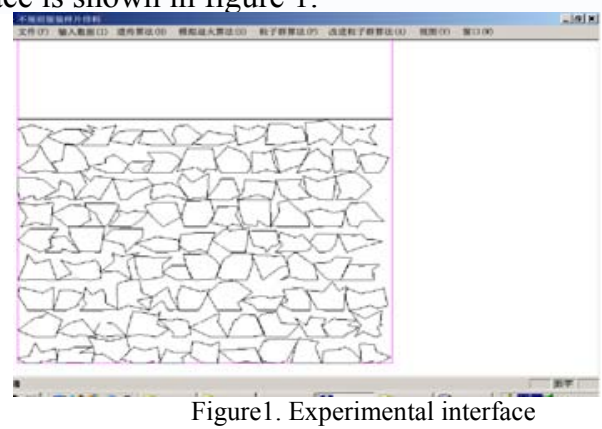

\section{B. Parameter settings}

Genetic algorithm: population set to a fixed value 300 ; iterative number is 100; the probability of crossover and mutation probability are floating value [6].

Simulated annealing algorithm: the initial temperature is 100 degrees, the cooling rate is 0.95 degrees / minutes, and end temperature of 0.001 degrees, the inner cycle number on each temperature remained consistent with the population of genetic algorithm, namely 300 .

Particle swarm algorithm: set the particle number is 300 , the iteration number is 100 .

Improved particle swarm optimization algorithm: set the particle number is 300 , the iteration number is 100 .

C. Marker making experiment results comparison and analysis

According to the above parameters, this experiment randomly generates 10 groups of irregular apparel plates for each algorithm, the marker making experimental results are in the following table:

TableI. The Test Results of Various Algorithms

\begin{tabular}{|c|c|c|c|c|}
\hline Algorithms & Population & $\begin{array}{l}\text { Iteration } \\
\text { number }\end{array}$ & $\begin{array}{l}\text { Average } \\
\text { utilization } \\
\text { rate of } \\
\text { fabric }\end{array}$ & $\begin{array}{l}\text { Average } \\
\text { running } \\
\text { time(second) }\end{array}$ \\
\hline Genetic algorithm & 300 & 100 & $60.23 \%$ & 235.6 \\
\hline $\begin{array}{l}\text { Simulated annealing } \\
\text { algorithm }\end{array}$ & 300 & 100 & $64.37 \%$ & 157. 3 \\
\hline $\begin{array}{l}\text { Particle } \\
\text { algorithm }\end{array}$ & 300 & 100 & $63.19 \%$ & 184. 1 \\
\hline $\begin{array}{l}\text { Improved particle } \\
\text { swarm optimization } \\
\text { algorithm }\end{array}$ & 300 & 100 & $68.04 \%$ & 159.5 \\
\hline
\end{tabular}

We can see from the test results in table I, under the same experimental conditions, the improved particle swarm algorithm can not only achieve the highest utilization rate of fabric, but also the running time is close to optimal, so we can judge that the improved particle swarm algorithm is a successful algorithm.

\section{CONCLUSION}

Intelligent optimizing marker making algorithm is core technology of apparel CAD, it plays a decisive role on the garment CAD system, this paper proposed an improved particle swarm optimization algorithm which is based on particle swarm algorithm, and the experiment results prove that this algorithm is effective and successful.

\section{ACKNOWLEDGMENT}

This research was supported by the funds from: Foundation item 1:Construction Funds of Master Degree Awarded Unit of Quanzhou Normal University; Foundation item 2: Fujian university key project construction service routines "The port logistics information platform construction, Haqixi prefecture",Project Number: A103.

\section{REFERENCES}

[1] D.F. Zhang,Y. Kang,A.s Deng, "A new heuristic recursive algorithm for the strip rectangular packing problem".Computer\&Operations Research 2006,pp:2209-2217.

[2] Hopper E,Turton B. "A genetic algorithm for a 2D industrial packing problem".Computerand Industrial Engineering,1999,pp:375-378.

[3] Z.X.Jia,G.F.Yin, "Two-dimensional irregular parts packing with genetic algorithm". Journal of computer-aided design \& Computer Graphics, 2002,PP:467-470.

[4] X.P.Wang,L.M.Cao, "Genetic algorithm theory and Application". Xi'an Jiao Tong University press,2001,pp:4-9.

[5] M.Li,C.F.Song, "The two-dimensional irregular parts packing problem by particle swarm algorithm". Journal of Jiangnan University (NATURAL SCIENCE EDITION),2005,pp:266-269.

[6] X.Liu,C.Y.Xu,"Irregular graphics prototype design for automatic layout". Journal of Huazhong University of Science and Technology (NATURAL SCIENCE EDITION),2002,pp:19-20. 